\title{
Virulent Mutants of Phage P22
}

\section{Physiological Analysis of P22 virB-3 and Its Component Mutations'}

\author{
MORLEY J. BRONSON² AND MYRON LEVINE \\ Department of Human Genetics, University of Michigan, Ann Arbor, Michigan 48104
}

Accepted October 27, 1971

\begin{abstract}
The virulent mutant of phage P22, virB-3, consists of two mutations: $K 5$, which maps in the $c_{2}$ repressor gene, and $V x$, which maps between $c_{2}$ and $c_{3}$. Although neither P22 $K \sigma$ nor P22 $V x$ is virulent, each of these mutants can express gene functions not expressed by other nonvirulent phages in the presence of prophage immunity. In mixed superinfection of a lysogen with $\mathrm{P} 22$ vir $B-8$, only a small fraction of the yield consists of $c^{+}, c_{1}$, or $c_{2}$ phage even though the virulent grows normally. This is the phenomenon of replication inhibition. In contrast, $\mathrm{P} 22 \mathrm{~K} 5$ and to a lesser extent, P22 V $x$ show escape from replication inhibition. However, neither P22 K 5 nor P22 Vx alone replicates extensively in a lysogen. These mutants complement in trans for phage DNA synthesis and phage production. This result indicates that both P22 $K 5$ and P22 Vx express some of the functions required for phage replication in immune conditions. In addition, P22 K5 kills lysogens at low multiplicities, and lysis is observed when these complexes are treated with chloroform.

P22 vir $B-3$ is repressed by the $c_{2}$ gene product made by a $c^{+}$phage in mixed infection of a sensitive host. There is an inverse relationship between the burst size and the multiplicity of infeetion of the $c^{+}$phage. $\mathrm{P} 22$ vir B-8 represses its own growth at high multiplicities even though the $K 5$ mutation maps in the $c_{2}$ gene and confers a clear plaque phenotype to phage P22. Introduction of a second $c_{2}$ mutation into the P22 vir B-9 genome abolishes this multiplicity effect. These findings demonstrate that P22 $\operatorname{vir} B-3$ is sensitive to its own repressor and to that of a coinfecting phage bearing a $c_{2}{ }^{+}$allele. We have called this effect multiplicity repression. The residual sensitivity of P22 virB-3 to repressor suggests that at least one of its component mutations is of the operator constitutive type.
\end{abstract}

\section{INTRODUCTION}

The temperate bacteriophage $\mathrm{P} 22$ is able to establish lysogeny in Salmonella typhimurium. Lysogens are immune to superinfection by phage P22 (Zinder, 1958) and have the potential to lyse and produce progeny of the prophage type on induction (Boyd, 1951). The integrated prophage also causes the appearance in the host cell of a

This work is supported by U.S. Public Health Service grants GM-15419-04 and NIH-5-T01GM00071 from the National Institute of General Medical Sciences.

2 Present address: Department of Biological Sciences, Stanford University, Stanford, California 94305 . new somatic antigen (Robbins and Uchida, 1962; Young et al., 1964) and exclusion of superinfecting genomes of P22 and heteroimmune phage (Rao, 1968; Walsh and Meynell, 1967). Maintenance of lysogeny is dependent upon functional products of the $c_{2}$ (Levine and Smith, 1964) and $m n t$ (Gough, 1968) genes.

Mutants of P22 have been isolated which produce phage progeny on superinfection of a P22 lysogen (Bronson and Levine, 1971). These virulent mutants have been classified into three groups: (1) VirA mutants, which map at or very near the mnt locus (Fig. 1); (2) VirB mutants, which contain two mutations mapping in the clear region; and (3) 


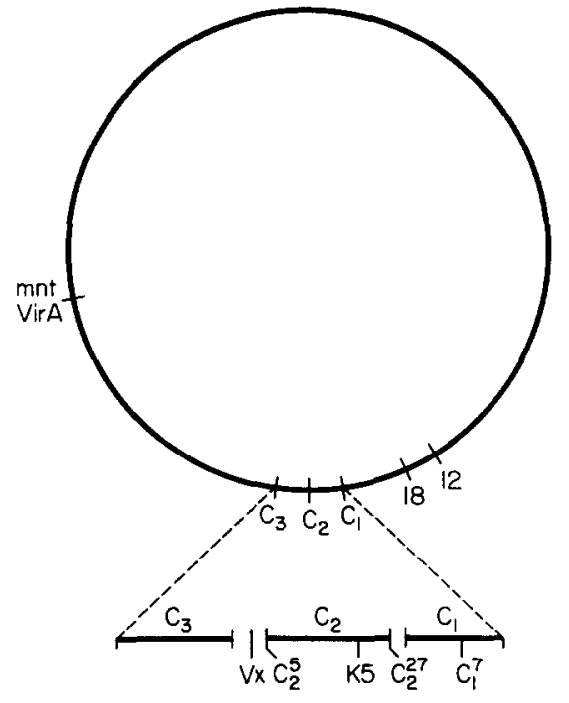

Fig. 1. Circular linkage map of phage P22 with clear region in detail.

VirC mutants, which are comprised of the determinants of both VirA and $\operatorname{Vir} B \mathrm{mu}$ tants. The $\operatorname{Vir} B$ mutant, $\mathrm{P} 22$ virB-3, has been analyzed in detail (Bronson and Levine, 1971). One of its component mutations, $V x$, maps between the $c_{2}$ and $c_{3}$ genes (Fig. 1). The other mutation, $K 5$, maps in the $c_{2}$ repressor gene (Fig. 1).

Phage P22 is sensitive to replication inhibition (Levine et al., 1970), first described for phages $\lambda$ and $\mathrm{P} 2$ by Thomas and Bertani (1964). In mixed superinfection of a lysogen by P22 virB-3 and a nonvirulent P22 $c_{1}$ mutant, virulent phage progeny represent greater than $98 \%$ of the yield. The burst size of the nonvirulent phage is less than its input. The P22 virB-3 DNA associates with the phage replication complex and undergoes normal replication and maturation, whereas little association of the input nonvirulent phage DNA with the complex is demonstrable (Botstein, 1968; Levine et al., 1970). The inability of the virulent mutant to complement nonvirulent DNA into the active replication machinery suggests that repressor exerts a direct physical effect in replication inhibition.

This report demonstrates that P22 K5 and to a lesser extent P22 $V x$ show escape from replication inhibition. In addition, both mutants express gene functions required for phage DNA replication in a lysogen. However, P22 vir $B-3$ is sensitive to high levels of $c_{2}$ repressor coded for by its own genome or by that of a coinfecting phage. These findings are discussed in relation to the mechanism of virulence of $\mathrm{P} 22$ virB-3.

\section{MATERIAIS AND METHODS}

Bacteria and phage. Strain 18, a derivative of Salmonella typhimurium LT2 cured of prophage PB1 (Zinder, 1958) was used as the sensilive host. Strain 210, which is strain 18 lysogenic for P22 sie1 ts2.1 ts12.1 (Rao, 1968) was used as the immune host. Prophage containing the sie mutation do not exclude superinfecting phage P22 (Rao, 1968; Walsh and Meynell, 1967). A galactose-negative strain was used as the indicator on EMB galactose agar plates (Levine, 1957). P22 virB-3 has a plating efficiency of 0.7 on the immune host relative to the sensitive host (Bronson and Levine, 1971). Nonvirulent P22 phage form plaques only on the sensitive host. The clear mutant phages P22 $c_{1}, \mathrm{P} 22 c_{2}$, and P22 $c_{3}$ have been described by Levine (1957). Phage bearing the mnt-1 mutation, originally called $v_{1}$ by Zinder (1958), are unable to establish stable lysogeny.

Media. L broth (Levine, 1957), supplemented M9 medium (Smith and Levine, 1964), soft agar for top layers (Levine, 1957), EMB galactose agar (Levine, 1957), indicator agar (Levine, 1957), and tryptone agar (Bronson and Levine, 1971) have been described.

Phage infections. Overnight cultures of the above strains were diluted $1: 100$ into $L B$ or supplemented M9 medium and grown to a concentration of $10^{8}$ cells $/ \mathrm{ml}$ with aeration at $37^{\circ}$. The log phase cells were infected at $37^{\circ}$ at various multiplicities. Superinfections of lysogenic strain 210 were performed at a total multiplicity of infection of 10 or less to avoid titration of repressor (Rao, 1968). After $5 \mathrm{~min}$ adsorption, the infected cells were diluted $1: 10$ in growth medium and antiserum $(K=2)$ for $5 \mathrm{~min}$ to inactivate unadsorbed phage. Samples were then diluted into growth tubes containing either LB or supplemented M9 medium. At 90 min after infection, chloroform was added to 
the growth tubes, and the lysates were assayed on the appropriate indicators. In one-step growth experiments, samples were assayed for phage at various times after infection. The burst size is defined as the number of plaque-forming phage particles produced at $90 \mathrm{~min}$ after infection divided by the number of infected cells. The frequencies of killing and of infectious center formation were determined by plating diluted samples of infected cells on EMB galactose agar plates at $10 \mathrm{~min}$ after infection. Surviving cells give rise to purple colonies, whereas infectious centers are identified as plaques.

Measurement of the rate of DNA synthesis. The rate of DNA synthesis was estimated by incorporation of $\left[{ }^{3} \mathrm{H}\right]$ thymidine into acidinsoluble material during a $1-\mathrm{min}$ pulse as described by Smith and Levine (1964).

\section{RESULTS}

\section{P22 VirB-3 Is Sensitive to Repressor}

P22 virB-3 shows normal kinetics of phage production on infection of either a sensitive or lysogenic host at $37^{\circ}$ at low multiplicities. The latent period is about $30 \mathrm{~min}$, and the burst size taken at $90 \mathrm{~min}$ after infection at a multiplicity of infection (m.o.i.) of 5, ranges around 100 (Table 1, infection 1; Table 2, infection 1). The prophage is not induced, suggesting that $\mathrm{P} 22$ virB-3 does not inactivate the prophage repressor.

The above data suggest that $\mathrm{P} 22$ virB-3 has a reduced sensitivity to repressor. If this is the case, higher levels of repressor than that made by a single prophage may inhibit development of P22 virB-3. This can be tested by coinfection of a sensitive host with P22 virB-3 and varying multiplicities of wild-type P22 $c^{+}$phage. If $\mathrm{P} 22 \operatorname{vir} B-3$ is sensitive to repressor, its burst size should decrease as the m.o.i. of P22 $c^{+}$is increased. Increasing the m.o.i. of a $c_{2}$ mutant which does not make repressor should not cause a reduction in burst size.

Sensitive strain 18 was infected with $\mathrm{P} 22$ vir $B-3$ at a constant multiplicity of 5 and a second phage at multiplicities ranging from 0 to 50 . As the m.o.i. of $\mathrm{P} 22 c^{+}$increased, the burst size of the mixed infection decreased (Table 1, infections 5-8). However, raising the m.o.i. of P22 $c_{2}{ }^{5}$ or P22 $c_{2}{ }^{27}$ did not decrease the burst size ('Table 1 , infections 9-12). Thus, P22 virB-3 phage development is sensitive to the $c_{2}$ repressor. Phage bearing the mnt-1 mutation make a defective mnt gene product resulting in inability to form stable lysogens (Zinder, 1958). Prophage bearing a temperaturesensitive mutation in the $m n t$ gene is induced if the temperature is raised (Gough, 1968). Thus, functional $m n t$ gene product is required for the maintenance of lysogeny and has properties characteristic of a repressor. Nevertheless, in mixed infection with P22 vir $B-3$, a decreased burst size was observed when the m.o.i. of $m n t-1$ was raised (Table 1 , infections 13 and 14). These results suggest that functional $m n t$ gene product is not required for inhibition of P22 virB-3. A decreased burst size with increased m.o.i. of phage supplying $c_{2}$ repressor will hereafter be referred to as mulitiplicity repression.

\section{VirB-3 Can Repress Its Own Development}

P22 virB-3 contains a mutation, $K 5$, which maps within the $c_{2}$ gene (Bronson and Levine, 1971). Both P22 virB-3 and P22 K5 make clear plaques on sensitive strain 18 , and P22 $K 5$ complements as a $c_{2}$ mutant (Bronson and Levine, 1971). It was, therefore, surprising that P22 virB-3 itself shows multiplicity repression. As the m.o.i. of P22 $\operatorname{vir} B-3$ was raised from 5 to 50 , the burst size decreased from 104 to 1.1 (Table 1, infections 1-4). Thus, P22 virB-3, a virulent phage containing a $c_{2}$ mutation, can inhibit its own development. These results suggest that $\mathrm{P} 22$ vir $B-3$ makes functional repressor, and represses itself in the same way it is repressed by $c^{+}$phage. Two possible explanations for this self-repression by $\mathrm{P} 22$ $\operatorname{vir} B-3$ were considered: (1) in the double mutant P22 virB-3, the $V x$ mutation in some way suppresses the defective $c_{2}$ phenotype expressed by the $K 5$ allele. (2) the $c_{2}$ gene of P22 K5 codes for a product with some repressor activity. At high multiplicities of infection, enough of this product is made to cause multiplicity repression. To distinguish between these hypotheses, the double mutant P22 $\mathrm{Vxc}_{2}{ }^{\mathbf{5}}$ and the single mutant P22 K5 were tested for multiplicity repression. 
TABLE 1

Multiplicity Repression in Sensitive Strain $18^{a}$

\begin{tabular}{|c|c|c|c|c|c|c|c|}
\hline Infection & Phage & M.o.i. & $\underset{\text { size }}{\text { Burst }}$ & Infection & Phage & M.o.i. & $\begin{array}{c}\text { Burst } \\
\text { size }\end{array}$ \\
\hline 1 & $\operatorname{vir} B-8$ & 5 & 104 & 15 & K5 & 5 & 63 \\
\hline 2 & & 10 & 34 & 16 & & 10 & 24 \\
\hline 3 & & 20 & 5.4 & 17 & & 20 & 5.7 \\
\hline 4 & & 50 & 1.1 & 18 & & 50 & 1.7 \\
\hline 5 & $\operatorname{vir} B-3 \times c^{+}$ & $5: 5$ & 47 & 19 & $v x c_{2}^{5}$ & 5 & 200 \\
\hline 6 & & $5: 10$ & 26 & 20 & & 50 & 99 \\
\hline 7 & & $5: 20$ & 7.4 & 21 & $V x c_{2}{ }^{5} K 5$ & 5 & 210 \\
\hline 8 & & $5: 50$ & 2.2 & 22 & & 50 & 75 \\
\hline 9 & $\operatorname{vir} B-3 \times c_{2}^{5}$ & $5: 5$ & 74 & 23 & $V x K \delta c_{2}^{27}$ & 5 & 275 \\
\hline 10 & & $5: 50$ & 140 & 24 & & 50 & 180 \\
\hline 11 & $\operatorname{vir} B-9 \times c_{2}^{27}$ & $5: 5$ & 89 & 25 & $V x c_{2}{ }^{5} K 5 \times c^{+}$ & $5: 5$ & 100 \\
\hline 12 & & $5: 50$ & 66 & 26 & & $5: 50$ & 2.4 \\
\hline 13 & $\operatorname{vir} B-3 \times m n t-1$ & $5: 5$ & 37 & 27 & $V x K 5 c_{2}^{27} \times c^{+}$ & $5: 5$ & 130 \\
\hline 14 & & $5: 50$ & 6.5 & 28 & & $5: 50$ & 1.9 \\
\hline
\end{tabular}

a All infections were performed at $37^{\circ}$. At 90 min after infection, the cultures were treated with chloroform and assayed for plaque-forming particles. The burst size is defined as the number of plaqueforming particles produced divided by the number of infected cells.

TABLE 2

\begin{tabular}{|c|c|c|c|}
\hline \multicolumn{4}{|c|}{$\begin{array}{l}\text { MULTIPLICITY REPRESSION IN LYSOGENIC } \\
\text { STRATN } 210^{\circ}\end{array}$} \\
\hline Infection & Phage & M.o.i. & Burst Size \\
\hline 1 & \multirow[t]{3}{*}{$\operatorname{vir} B-s$} & 5 & 82 \\
\hline 2 & & 10 & 31 \\
\hline 3 & & 50 & 4.5 \\
\hline 4 & \multirow[t]{2}{*}{$\operatorname{virB-3} \times c^{+}$} & $5: 5$ & 94 \\
\hline 5 & & $5: 50$ & 68 \\
\hline 6 & \multirow[t]{2}{*}{$\operatorname{vir} B-3 \times V x$} & $5: 5$ & 45 \\
\hline 7 & & $5: 50$ & 6.9 \\
\hline 8 & \multirow[t]{2}{*}{$\operatorname{vir} B-3 \times K 5$} & $5: 5$ & 73 \\
\hline 9 & & $5: 50$ & 9.7 \\
\hline 10 & \multirow[t]{2}{*}{$\operatorname{vir} B-3 \times V x c_{2}^{5}$} & $5: 5$ & 100 \\
\hline 11 & & $5: 50$ & 77 \\
\hline
\end{tabular}

${ }^{a}$ All infections were performed at $37^{\circ}$. At 90 min after infection, the cultures were treated with chloroform and assayed for plaque-forming particles. The burst size is defined as the number of plaque-forming particles produced divided by the number of infected cells.

If the $V x$ mutation suppresses the $K 5$ phenotype, the single P22 K5 mutant should not exhibit multiplicity repression. P22 $V x c_{2}{ }^{5}$ would be expected to exhibit self-repression at high multiplicities if $V x$ suppression is gene specific. If, on the other hand, phage carrying the single $K 5$ mutation make re- pressor, then $\mathrm{P} 22 \mathrm{~K} 5$ should show multiplicity repression, whereas $\mathrm{P} 22 V \mathrm{Vc}_{2}$ should not. The data clearly show that infection by P22 K5 resulted in multiplicity repression to about the same extent as observed with P22 virB-8 (Table 1, infections 15-18). Multiplicity repression was not observed in the infections by P22 $V x c_{2}{ }^{5}$ (Table 1, infections 19-20). These findings suggest that $K 5$ is a mutation in the $\epsilon_{2}$ gene which confers a clear plaque phenotype to phage P22 but does not eliminate all $c_{2}$ repressor activity.

Since neither $\mathrm{P} 22 c_{2}{ }^{5}$ nor P22 $c_{2}{ }^{27}$ cause multiplicity repression, introduction of either the $c_{2}{ }^{5}$ or the $c_{2}^{27}$ mutation in cis to $K 5$ would be expected to result in destruction of any repressor activity and thereby abolish multiplicity repression. To test this, the triple virulent mutants $\mathrm{P} 22 \quad V x c_{2}{ }^{5} \mathrm{~K} 5$ and $\mathrm{P} 22$ $V x K 5 c_{2}{ }^{27}$ were constructed. Neither of these phages in single infection showed the multiplicity repression characteristic of P22 K5 and P22 virB-3 (Table 1, infections 21-24). However, both P22 Vxc. ${ }^{5} \mathrm{~K} 5$ and P22 $V x K 5 c_{2}{ }_{2}^{27}$ were repressed in mixed infections with high multiplicities of wild-type $c^{+}$ phage (Table 1, infections 25-28). These results demonstrate that the introduction of an additional $c_{2}$ mutation in cis can abolish the self-repression of $\mathrm{P} 22 \operatorname{vir} B-3$, 
and that the resultant triple virulent mutants are still sensitive to multiplicity repression induced by a second phage.

\section{Multiplicity Repression in a Lysogen}

P22 virB.9 undergoes multiplicity repression in single superinfection of lysogenic strain 210 (Table 2, infections 1-3). However, mixed superinfection of $\mathrm{P} 22$ virB-3 and wild-type P22 $c^{+}$does not result in multiplicity repression when the m.o.i. of the latter phage is increased (Table 2, infections 4 and 5). This is in striking contrast to mixed infection of the sensitive host by these phayes (Table 1 , infections 5-8) and indicates that wild-type phage do not contribute sufficient repressor to inhibit development of $\mathrm{P} 22$ virB-3.

In contrast to wild-type phage, both P22 $V x c^{+}$and $\mathrm{P} 22 \mathrm{~K} 5$ caused multiplicity repression upon mixed superinfection of the lysogenic host with $\mathrm{P} 22$ virB-3 (Table 2, infections 6-9). P22 $\operatorname{Vxc}_{2}{ }^{5}$, a phage which does not make functional $c_{2}$ gene product did not repress P22 virB-3 (Table 2, infections 10 and 11 ). These results indicate that P22 $V x c^{+}$and P22 K5 produce repressor in the lysogenic host, and suggest that this property is associated with the constitutive nature of the $V x$ and $K 5$ mutations.

\section{Escape from Replication Inhibition}

The inability of wild-type P22 $c^{+}$to cause multiplicity repression in a lysogen may be a consequence of replication inhibition. In mixed superinfections of lysogenic strain 210 with $\mathrm{P} 22$ virB-s and either wild-type $\mathrm{P} 22 c^{+}, c_{1}$, or $c_{2}$ phage at an m.o.i. of 5 each, the nonvirulent phage represented only a few percent of the phage yield in each case (Table 3 , infections $2,4,6$, and 8 ). The burst sizes of the nonvirulent phages in mixed superinfection with P22 virB-3 showed little or no increase over the burst sizes observed on single superinfection (Table 3 , infections 1-8). Progeny of the prophage type represented less than $0.5 \%$ of the phage yield for each superinfection. These results demonstrate that the nonvirulent phages are repressed even though the virulent phage replicated normally in a lysogen. When these phages mixedly infected strain 18 at the same multiplicities, in each case over $25 \%$ of the phage yield consisted of the nonvirulent phage (Table 3 , infection 1-8), ruling out competition with the virulent phage as the reason for the low yields of nonvirulents in the lysogenic host.

Like $c^{+}, c_{1}$, and $c_{2}$ phages, P22 $K 5$ is nonvirulent and produces a burst size that is large on the sensitive host and small on the lysogenic host (Table 3, infection 9). However, in mixed superinfection of strain 210 with $\mathrm{P} 22$ virB-3, P22 K5 represented $44 \%$ of the phage yield (Table 3 , infection 10), a greater than 15 -fold increase in burst size over that found on single superinfection. This burst size of P22 K5 was equal to that observed in mixed infection of the sensitive host. We conclude from these data that P22 $K 5$ escapes replication inhibition.

P22 $\mathrm{Vxc}^{+}$and the double mutants P22 $V x c_{2}{ }^{5}$ and P22 $V x c_{2}{ }^{27}$ also gave small burst sizes on the lysogenic host (Table 3 , infections 11, 13, and 15). In mixed superinfection with P22 virB-3 the burst size of each of these mutants was increased (Table 3 , infections 11-16). The burst size and percent of yield of P22 $V x c_{2}{ }^{5}$ and P22 $V x c_{2}{ }^{27}$ in mixed superinfections was about three times that of P22 $c_{2}{ }^{5}$ and P22 $c_{2}{ }^{27}$ in the corresponding superinfections (Table 3 , ef. infection 12 with infection 6 and infection 14 with infection 8). However, the percentages of P22 $V x c_{2}{ }^{5}$ and P22 $V x c_{2}{ }^{27}$ in the yields from mixed superinfection of the lysogenic host are still much less than those found in lysates of mixed infections of the sensitive host. The burst size of P22 $\mathrm{Vxc}^{+}$was 10 -fold greater in mixed superinfection than in single superinfection (Table 3 , cf. infection 16 with infection 15) and three times greater than that found for wild-type P22 $c^{+}$in the corresponding mixed superinfection (Table 3 , cf. infection 16 with infection 2). We conclude that the $V x$ mutation has a small effect on relieving replication inhibition of a nonvirulent phage.

The capacity to cause multiplicity repression in the lysogenic host is correlated with escape from replication inhibition. The $V x$ and $K 5$ mutations appear to confer both of these properties simultaneously to phage 
TABLE 3

Replication Inhibition with Phage P22a

\begin{tabular}{|c|c|c|c|c|c|c|c|}
\hline \multirow[b]{2}{*}{ Infection } & \multirow[b]{2}{*}{ Phage } & \multicolumn{3}{|c|}{ Sensitive Strain 18} & \multicolumn{3}{|c|}{ Lysogenic Strain 210} \\
\hline & & $\begin{array}{c}\text { Total } \\
\text { burst size }\end{array}$ & $\begin{array}{c}\text { Nonvirulent } \\
\text { burst size }\end{array}$ & $\begin{array}{c}\% \\
\text { Nonvirulent }\end{array}$ & $\begin{array}{c}\text { Total } \\
\text { burst size }\end{array}$ & $\begin{array}{c}\text { Nonvirulent } \\
\text { burst size }\end{array}$ & $\begin{array}{c}\% \\
\text { Nonvirulent }\end{array}$ \\
\hline 1 & $c^{+}$ & 90 & & & 0.4 & & \\
\hline 2 & $c^{+} \times \operatorname{vir} B-s$ & 45 & 12 & 27 & 94 & 2.2 & 2.3 \\
\hline 3 & $c_{1}{ }^{7}$ & 200 & & & 2.6 & & \\
\hline 4 & $c_{1}^{7} \times \operatorname{vir} B-3$ & 96 & 40 & 42 & 90 & 1.4 & 1.5 \\
\hline 5 & $c_{2}^{5}$ & 205 & & & 3.3 & & \\
\hline 6 & $c_{2}{ }^{5} \times \operatorname{vir} B-3$ & 74 & 28 & 38 & 110 & 2.4 & 2.2 \\
\hline 7 & $c_{2}^{27}$ & 104 & & & 1.1 & & \\
\hline 8 & $c_{2}^{27} \times \operatorname{vir} B-3$ & 89 & 42 & 47 & 74 & 4.5 & 6.1 \\
\hline 9 & $K 5$ & 93 & & & 2.0 & & \\
\hline 10 & $K 5 \times \operatorname{vir} B-3$ & 84 & 32 & 38 & 73 & 32 & 44 \\
\hline 11 & $V x c_{2}^{5}$ & 230 & & & 2.5 & & \\
\hline 12 & $V x c_{2}{ }^{5} \times \operatorname{vir} B-3$ & 98 & 37 & 37 & 100 & 7.3 & 7.3 \\
\hline 13 & $V x c_{2}^{27}$ & 235 & & & 3.6 & & \\
\hline 14 & $V x c_{2}^{27} \times \operatorname{vir} B-3$ & 69 & 40 & 58 & 98 & 16 & 16 \\
\hline 15 & $V x c^{+}$ & 15 & & & 0.6 & & \\
\hline 16 & $V x c^{+} \times \operatorname{virB-3}$ & 9.5 & 1.4 & 15 & 45 & 6.9 & 13 \\
\hline
\end{tabular}

a Single infections werc performed at an m.o.i. of 5 , mixed infections at an m.o.i. of 5 for each phage. The clear plaques with a small ring of surviving bacteria in the center characteristic of $\operatorname{vir} B-3$ are easily distinguishable from turbid plaques made by $c^{\dagger}$ phage and the completely clear plaques made by $K \bar{b}$, $c_{1}$, and $c_{2}$ phage.

$\mathrm{P} 22$. In contrast, wild-type $\mathrm{P} 22 c^{+}$is replication inhibited and does not cause multiplicity repression in a lysogen.

\section{$K 5$ and $V x$ Complement in a Lysogen}

When the $K 5$ and $V x$ mutations are in the cis position, as in the double mutant P22 vir $B-3$, good phage yields result on superinfection of a lysogenic host at low multiplicities. We asked whether the $K 5$ and $V x$ mutations in the trans position could promote phage production in a lysogen. Although neither P22 K5 nor P22 Vx alone produced significant bursts on superinfection of the lysogenic strain 210 (Table 4, infections 1-4), mixed superinfection by these phages resulted in phage yields at the level found for P22 virB-3 (ef. Table 4, infections 5-7 with Table 2 , infections 1 and 2). Virulent recombinants represented less than $1 \%$ of the phage yield.

In mixed infection of sensitive strain 18 by $K 5$ and $V x$, the P22 $V x$ markers represented approximately half the phage yield (Table 4). However, mixed superinfection of the lysogenic host under the same conditions resulted in only about $20 \%$ of the phage yield carrying the $V x$ markers (Table 4). This result is consistent with the finding that P22 K5 shows complete excape from replication inhibition, whereas P22 Vx shows only partial excape.

A number of $c_{1}, c_{2}$, and $c_{3}$ mutants were tested to see whether they could complement either P22 $K 5$ or P22 $V x$ in a lysogen and were not able to do so. 'These data indicate that $K 5$ and $V x$ are specific mutations in the clear region which confer to phage P22 constitutive expression of diffusible gene products necessary for growth.

\section{DNA Synthesis in Superinfected Lysogens}

Superinfection of lysogenje strain 210 by P22 virB-3 resulted in a rate of DNA synthesis pattern (Fig. 2A) similar to that found by Smith and Levine, 1964) for P22 $c_{1}$ infection of a sensitive host. There was a brief drop in the rate of DNA synthesis immediately after infection followed by a rapid increase in rate above the level of the uninfected control, reaching a peak at about 20 minutes after superinfection. The rate of 
TABLE 4

Complementation in a Lysogen betwein P22 K5 and P22 V $x^{a}$

\begin{tabular}{|c|c|c|c|c|c|c|c|}
\hline \multirow[b]{2}{*}{ Infection } & \multirow[b]{2}{*}{ Phage } & \multicolumn{3}{|c|}{ Sensitive strain 18} & \multicolumn{3}{|c|}{ Lysogenic strain 210} \\
\hline & & $\begin{array}{c}\text { Total } \\
\text { burst size }\end{array}$ & $\begin{array}{l}V x \text { burst } \\
\text { size }\end{array}$ & $\begin{array}{l}\% V x \\
\text { markers }\end{array}$ & $\begin{array}{c}\text { Total } \\
\text { burst size }\end{array}$ & $\begin{array}{l}V x \text { burst } \\
\text { size }\end{array}$ & $\begin{array}{l}\% V x \\
\text { marker }\end{array}$ \\
\hline 1 & $K 5$ & 24 & & & 2.2 & & \\
\hline 2 & $V x c^{+}$ & 1.7 & & & 1.2 & & \\
\hline 3 & $V x c_{2}^{2 \pi}$ & 230 & & & 3.9 & & \\
\hline 4 & $V x c_{2}^{5}$ & 170 & & & 4.4 & & \\
\hline 5 & $K 5 \times V x c^{+}$ & 10 & 4.2 & 42 & 34 & 6.9 & 19 \\
\hline 6 & $K 5 \times V_{x c_{2}^{27} h_{21}}$ & 56 & 29 & 52 & 44 & 9.2 & 21 \\
\hline 7 & $K 5 h_{21} \times V x c_{2}^{5}$ & 38 & 19 & 49 & 18 & 4.0 & 22 \\
\hline
\end{tabular}

"The total m.o.i. in all infections was 10 . In mixed infections the m,o.i. was 5 for each phage. The large clear plaques made by $\mathrm{P} 22 \mathrm{~K} 5$ are easily distinguishable from the small turbid plaques made by P22 $V x c^{+}$. To distinguish between P22 $K 5$ and P22 Vxc ${ }_{2}^{5}$ or P22 $V x c_{2}^{27}$, one of the parental phage carried the $h_{21}$ plaque morphology marker, which confers no selective advantage or disadvantage.

DNA synthesis then declined sharply as the bacteria lysed and phage progeny were released.

Although P22 K5 is not subject to replication inhibition, superinfection of strain 210 by $\mathrm{P} 22 K 5$ did not lead to a greatly increased rate of DNA synthesis. The rate of DNA synthesis at no time exceeded the rate in the uninfected control (Fig. 2B). P22 $V x c_{2}{ }^{27}$ showed a pattern of DNA synthesis similar to that of P22 $K 5$ on superinfection of the lysogenic host (Fig. 2C). Mixed superinfection by $\mathrm{P} 22 \mathrm{~K} 5$ and $\mathrm{P} 22 \mathrm{Vxc}_{2}{ }^{27}$ resulted in a rate of DNA synthesis pattern similar to P22 virB-3 (Fig. 2D). Since P22 K5 and P22 $V x$ complement to make DNA in a lysogen, these results indicate that diffusible products controlled at both the $K 5$ and $V x$ mutational sites are necessary for extensive phage DNA replication.

\section{Cell Killing and Lysis of a Lysogen}

When lysogenic strain 210 was superinfected by $\mathrm{P} 22$ virB-3 at an m.o.i. of 5 , about $98 \%$ of the cells were killed. The optical density of the culture increased until $30 \mathrm{~min}$ after superinfection, at which time lysis occurred (Fig. 3). Strain 210 was superinfeeted with $\mathrm{P} 22 c_{2}{ }^{5}, \mathrm{P} 22 V x c_{2}{ }^{5}$ or $\mathrm{P} 22 \mathrm{~K} 5$ at an m.o.i. of 5 to determine the effect of the individual mutations on cell killing and lysis. Less than $1 \%$ of the cells superinfected by $\mathrm{P} 22 c_{2}{ }^{5}$ were killed. The optical density increased with time at the same rate as the uninfected control (Fig. 3). Supcrinfection by $\mathrm{P} 22 V x c_{2}{ }^{5}$ resulted in a similar increase in optical density of the culture, and less than $4 \%$ of the cells were killed (Fig. 3). In contrast, superinfection by $\mathrm{P} 22 K 5$ killed $46 \%$ of the cells. The optical density of the culture increased until $30 \mathrm{~min}$ after superinfection, after which time the optical density remained approximately constant until $60 \mathrm{~min}$ after superinfection (Fig. 3). Between 60 and $90 \mathrm{~min}$, the optical density increased slightly. Although $46 \%$ of the cells were killed, only $3 \%$ gave rise to infectious centers, and the burst size was only 2.0. When the P22 K5 superinfected culture was treated with chloroform at $90 \mathrm{~min}$, the optical density dropped, giving the appearance of cell lysis. In contrast, cells superinfected by $\mathrm{P} 22 c_{2}{ }^{5}$ or P22 $V x_{2}{ }^{5}$ gave no indication of lysis when treated with chloroform. These results indicate that the $K 5$ mutation but not the $V x$ mutation confers the ability to kill upon superinfection of a lysogen. Furthermore, since lysogens superinfected by $\mathrm{P} 22 \mathrm{~K} 5$ are lysed by treatment with chloroform, it is likely that the $K 5$ mutation confers constitutive expression of some late gene function(s).

\section{DISCUSSION}

The ability of P22 virB-3 to grow in an immune host without inducing prophage indicates that its component mutations, $K 5$ 

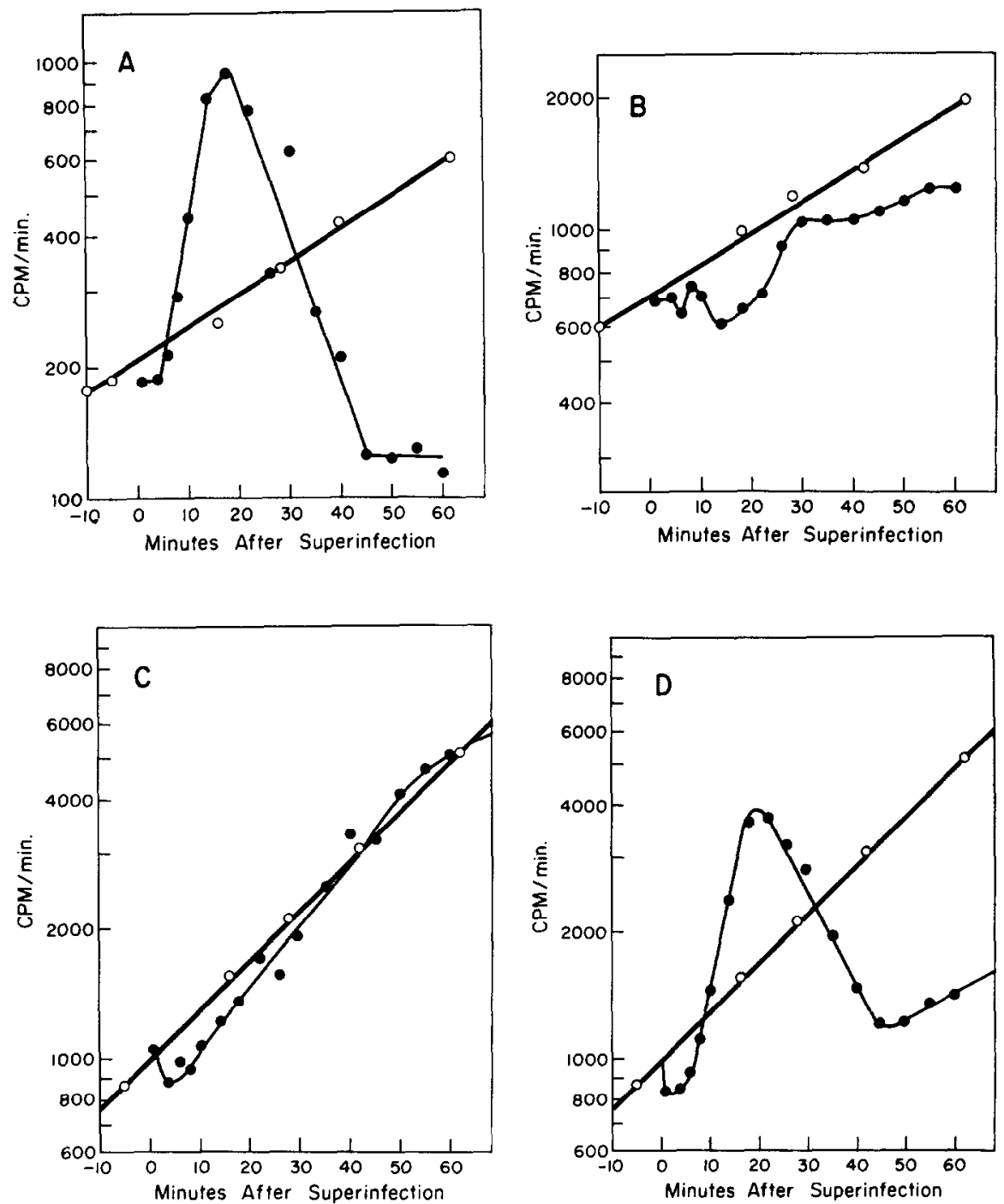

FIG. 2. Rate of incorporation of $\left[{ }^{3} \mathrm{H}\right]$ thymidine into DNA after superinfection of lysogenic strain 210 at $37^{\circ} \mathrm{C}$. One-minute pulses of $\left[{ }^{3} \mathrm{H}\right]$ thymidine were administered as described by Smith and Levine (1964). (A) $\mathrm{P} 22$ virB-3, m.o.i. $=5$. (B) $\mathrm{P} 22 K 5$, m.o.i. $=5$. (C) $\mathrm{P} 22 V x c_{2}^{27}$, m.o.i. $=5$. (D) $\mathrm{P} 22 K 5$, m.o.i. $=5+$ P22 $V x c_{2}{ }^{27}$, m.o.i. = 5. O-O, Uninfected control culture; -O, superinfected cells.

and $V x$, relieve this phage of repressor control. The constitutive nature of the $K 5$ and $V x$ mutations was demonstrated in several ways:

1. Although neither P22 K5 nor P22 Vx replicates extensively in a lysogen, they complement in trans for phage DNA synthesis and production of progeny phage. We conclude that both P22 K5 and P22 Vx can express functions involved in replication of phage P22 in the presence of repressor.
2. P22 K5 and, to a lesser extent, P22 Vx escape replication inhibition. This cis function is not expressed by other nonvirulent phage.

3. Superinfection by $\mathrm{P} 22 K \delta$ results in killing of lysogens and expression of some late gene function(s). These effects have not been demonstrated for P22 Vx.

The constitutive expression of phage functions by P22 virB-3 is not absolute. Growth of $\mathrm{P} 22$ vir $B-3$ is increasingly inhibited as the 


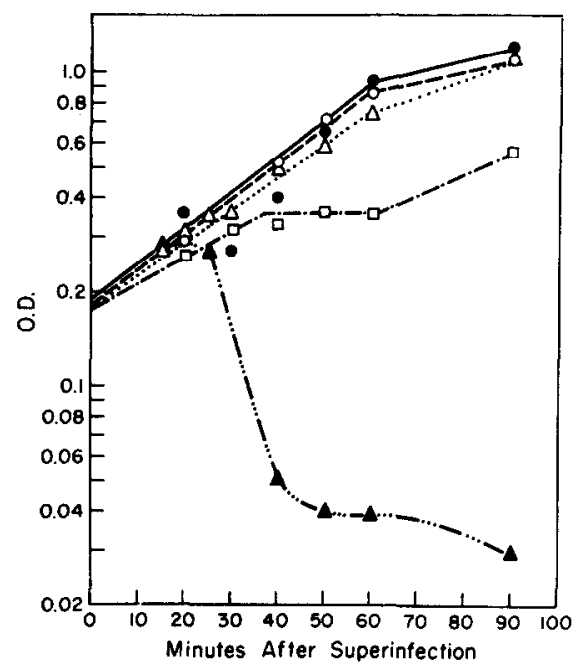

FIg. 3. Effect of superinfection of strain DB103 on cell growth as measured by increase in optical density (O.D.). Log phase cultures of the lysogenic host were superinfected at an m.o.i. of 5 , shaken at $37^{\circ}$, and periodically assayed for O.D O..--- $O$, uninfected control; $\mathbf{\Delta} \cdot-\boldsymbol{A}$, P22 virB-3; $-\mathbf{-}$, P22a $c_{2}{ }^{5} ; \triangle \cdots \triangle \triangle$, P22 $V x c_{2}{ }^{5} ; \square-\cdot-\square$, P22 K5.

multiplicity of coinfecting wild-type phage is raised. We have given the name multiplicity repression to the phenomenon of a decreased burst size with increased multiplicity. Multiplicity repression is due to the action of the $c_{2}$ repressor and is not dependent on a functional $m n t$ gene product.

P22 virB-3 also exhibits multiplicity repression in single infection. 'This finding was unexpected, since one of its component mutations, $K 5$, maps in the $c_{2}$ gene. Introducing either the $c_{2}{ }^{5}$ or $c_{2}{ }^{27}$ mutation in cis to P22 virB-3 eliminates self-induced multiplicity repression. This indicates that the $K 5$ mutation does not completely destroy $c_{2}$ repressor activity. We conclude that P22 virB-3 synthesizes active $c_{2}$ repressor and exhibits a reduced but finite sensitivity to the action of this repressor.

Two $c_{2}$ mutants other than P22 K5 also exhibit multiplicity repression (unpublished data). By this test we can classify P22 $c_{2}$ mutants into two groups: those which retain some repressor activity and those which have no detectable repressor function. More data must be accumulated to determine a possible relationship between mutational site and multiplicity repression. However, it should be noted that the two $c_{2}$ mutations, $c_{2}{ }^{5}$ and $c_{2}{ }^{27}$, shown to destroy all repressor activity define the $c_{\text {, gene by mapping at its }}$ extremes (Levine and Curtiss, 1961).

Multiplicity repression resembles a multiplicity effect described for phage P22 in which reduction and the lysogenic response are favored by high multiplicities of wildtype P'22 $c^{+}$phage (Boyd, 1951; Levine, 1957), but it is unclear whether or not the two effects are mediated by the same mechanism. In this context it should be noted that $\mathrm{P} 22$ virB-3 does not form stable lysogens (unpublished data). Clear mutants of the closely related heteroimmune phage $\mathrm{L}$ have been described which show an effect similar to multiplicity repression (Bezdek et al., 1970).

The ability of P22 $K 5$ and P22 $V x$ to express gene functions in an immune host and the sensitivity of $v i r B-3$ to $c_{2}$ repressor give some clues as to the nature of the $K 5$ and $V x$ mutations. The ability to complement in trans suggests that P22 K5 and P22 $V x$ are constitutive for two different segments of the P22 genome normally under repressor control, and may be analogous to the mutations involved in phage $\lambda$ virulence. The lambda $c$ I repressor, analogous to the $\mathrm{P} 22 c_{2}$ repressor, blocks transcription of the $\lambda$ genome by binding to two operator sites which map on each side of the $c \mathrm{I}$ gene and control the adjacent operons (Ptashne and Hopkins, 1968; Taylor et al., 1967). A virulent $\lambda$ mutant bearing operator constitutive $\left(O^{c}\right)$ mutations at both of these sites has been described (Ptashne and Hopkins, 1968). These $O^{c}$ mutations, $v_{2}$ in the left-hand operator and $v_{1} v_{3}$ in the righthand operator, decrease but do not abolish the affinity of $\lambda$ DNA for repressor in vitro (Ptashne and Hopkins, 1968). Sly and Rabideau (1969) suggested that residual repressor binding to $\lambda v_{2}$ DNA prevents fully constitutive expression of the adjacent operon. P22 virB-3 may be sensitive to multiplicity repression for a similar reason. $V x$ maps to the left of the $c_{2}$ gene, and $K 5$ maps in $c_{2}$ to the right of $V x$. If $K 5$ and/or $V x$ are $O^{c}$ mutations leaving $\mathrm{P} 22 \mathrm{DNA}$ with some residual affinity for repressor at the 
operator sites, high concentrations of the $c_{2}$ gene product could create a binding equilibrium favoring repression. It should be stressed that only one of the component mutations of $\mathrm{P} 22$ virB-3 need be an $O^{\circ}$ mutation of this type for multiplicity repression to obtain. The other mutation may be of the new promoter type as has been suggested for the $c_{17}$ mutation which is a component of a virulent mutant of phage $\lambda$ (Sly and Rabideau, 1969). $c_{17}$ maps at some distance from the $\lambda$ operator sites and creates constitutivity of the right-hand operon, suggesting release from operator control.

Escape from replication inhibition is a prerequisite for phage development in the presence of repressor. It follows that at least one component mutation should confer this cis property to $\mathrm{P} 22$ vir $\mathrm{B}-3 . \mathrm{P} 22 \mathrm{~K}$ i completely escapes from replication inhibition, whereas P22 $V x$ gives only partial escape. It has been demonstrated that the physical basis of replication inhibition is the direct block by prophage repressor on the association of input nonvirulent DNA with the P22 replication complex (Levine et al., 1970). This complex, called intermediate $I$ by Botstein (Botstein, 1968), consists of parental phage DNA, newly synthesized DNA, and other cell constituents. P22 K5 genomes, in accord with their escape from replication inhibition, associate with intermediate I in a lysogen to a greater extent than other nonvirulent genomes (unpublished data). Although this association is dependent on the trans function of gene 25 (Levine et al., 1970 ), a cis function is required in addition. Three hypotheses are advanced to explain this cis function: (1) The site on the phage genome for binding of phage DNA to the replication machinery is blocked by bound repressor. Loss of affinity for repressor as a consequence of an $O^{\mathrm{c}}$ mutation would result in escape from replication inhibition. (2) Binding to intermediate $I$ is a consequence of general transeription. Constitutive transcription by a genome bearing an $O^{c}$ or a new promoter mutation would be required for association with intermediate $\mathrm{I}$ in a lysogen. (3) Transcription of a critical region of the input genome is required for binding to the replication machinery as has been suggested for phage lambda (Dove et al., 1969). The mechanisms suggested in (1) and (3) require events at unique sites on the P22 genome. Although both $K 5$ and $V x$ have effects on replication inhibition, these mutations map at different loci (Fig. 1). Therefore, mechanism 1 requires the assumption that there is more than one site of binding to intermediate I on the P22 genome. Mechanism 3 requires the assumption that both $K 5$ and $V x$ mutations confer constitutive transcription of a critical region of the genome. No further assumptions are required for mechauism 2. The $K \sigma$ mutation which confers complete escape from replication inhibition to the P22 genome may be either a new promoter or an $O^{\circ}$ mutation which is insensitive to repressor. The resultant strong transcription of the constitutive operon could lead to binding of the P22 $K 5$ genome to the replication complex. The partial sensitivily of P22 $V x$ to replication inhibition could be due to residual binding of repressor at the $V x$ site. $V x$ might be a leaky $O^{c}$ or a weak new promoter mutation which confers weakly constitutive transcription to the genome.

Mutations which confer escape from replication on phage $\lambda, v_{1} v_{3}$ and $c_{17}$ (Ptashne and Hopkins, 1968; Sly and Rabideau, 1969) have additional similarities to $K 5$. Two genes required for phage DNA synthesis map to the right of each mutation: genes 18 and 12 of phage P22 (Levine and Schott, 1971) and genes $O$ and $P$ at analogous positions in phage $\lambda$ (Sly and Rabideau, 1969). Like $\lambda v_{1} v_{3}$ and $\lambda c_{17}$ (Sly and Rabideau, 1969), P22 KJ shows constitutive killing. Despite these similarities, the $\lambda$ mutants have some properties not associated with P22 K5. Both $\lambda v_{1} v_{3}$ and $\lambda c_{17}$ are constitutive for genes $O$ and $P$, although transcription of genes to the left of $\mathrm{cI}$ is under normal repressor control (Sly and Rabideau, 1969). These $\lambda$ mutants replicate extensively in a lysogen, suggesting that $O$ and $P$ functions are sufficient for $\lambda$ phage DNA synthesis. Strong virulent mutants are formed when a $c \mathrm{I}$ mutation is introduced in $c i s$ to $v_{1} v_{3}$ or $c_{17}$. In contrast to these $\lambda$ mutants, introduction of a mutation in the repressor gene in cis to 
$K 5$ does not result in the formation of a virulent mutant (Bronson and Levine, 1971). Furthermore, P22 K5 alone does not replicate extensively in an immune host. A function(s) supplied by P22 $V x$ is required for phage DNA synthesis. If by analogy with phage $\lambda$ we assume that the products of genes 18 and 12 are sufficient for replication of phage P22, it appears that $\mathrm{P} 22 K 5$ is not constitutive for these genes. This would suggest that a constitutive function supplied by $\mathrm{P} 22 V x$ can activate $\mathrm{P} 22 K 5$ to transcribe genes 18 and 12 in the presence of repressor. Alternatively, $\mathrm{P} 22 \mathrm{~K} 5$ alone may be constitutive for 18 and 12 , but the functions of these genes are not sufficient for extensive phage replication. The constitutive function(s) supplied by P'2 $V x$ may be required in addition.

The inability of wild-type $c^{+}$phage to cause multiplicity repression of $\mathrm{P} 22$ virB-3 in a lysogen suggests that the superinfecting replication inhibitcd genome cannot express the $c_{2}$ gene function in the presence of immunity. Both $\mathrm{P} 22 K 5$ and $\mathrm{P} 22 V x$ induce multiplicity repression in a lysogen and escape from replication inhibition. This correlation between multiplicity repression and escape from replication inhibition suggests that superinfecting genomes must associate with the $\mathrm{P} 22$ replication complex in order to transcribe the $c_{2}$ gene. Wild-type $\mathrm{P} 22 c^{+}$inhibits growth of VirA mutants in a lysogen, suggesting that they can synthesize repressor (Swanson and Botstein, personal communication). This result is complicated by the apparent ability of VirA mutants to inactivate or inhibit synthesis of the prophage repressor. The amount of repressor synthesized by $\mathrm{P} 22 c^{+}$in a lysogen may be sufficient to repress $V i$, $A$ but not $\operatorname{Vir} B$ mutants. Although $\lambda c^{+}$phage are subject to replication inhibition, the $c \mathrm{I}$ repressor is expressed by $\lambda c^{+}$in a lysogen and inhibits the growth of a virulent mutant (Sly and Rabideau, 1969). It is unclear just how much repressor, if any, is synthesized by wild-type phage P22 in mixed superinfection with P22 virB-3.

In summary, we have demonstrated that the $K 5$ and $V x$ mutations confer virulence to P22 virB-3 by reducing the sensitivity of P22 DNA to the prophage repressor. P22 vir $B-3$ remains sensitive to high levels of the $c_{2}$ repressor and has the unusual property among virulent mutants of being able to repress its own development at high multiplicities. Both P22 K5 and P22 Vx express functions involved in phage DNA synthesis in the presence of repressor. In addition, P22 K5 is constitutive for cell killing and some late function(s).

\section{REFERENCES}

Brzofk, M., Soski, .J., and Amati, P. (1970). Properties of P22 and a related Salmonella typhimurium phage. Virology 40, 505-513.

Botstein, D. (1968). Synthesis and maturation of phage P22 DNA. I. Identification of intermediates. J. Mol. Biol. 34, 621-641.

Boyd, J. S. K. (1951) Observations on the relationship of symbiotic and lytic bacteriophage. J. Pathol. Bacteriol. 63, 445-457.

Bronson, M. J., and Levine, M. (1971). Virulent mutants of phage P22. I. Isolation and genetic analysis. J. Virol. 5, 559-568.

Dove, W. F., Hargrove, E., Ohashi, M., Haughi, F., and GUнA, A. (1969). Replicator activation in lambda. Jap. J. Genet. 44, 11-22.

Govgh, M. (1968). Second locus of bacteriophage P22 necessary for maintenance of lysogeny. $J$. Virol. 2, 992-998.

Levine, M. (1957). Mutations in the temperale phage P22 and lysogeny in Salmonella. Virology $3,22-41$.

Levine, M., Chakravorty, M., and Bronson, M. J. (1970). Control of the replication complex of bacteriophage P22. J. Virol. 6, 400-405.

Levine, M., and Curtiss, R. (1961). Genetic fine structure of the $c$ region and the linkage map of phage P22. Genetics 46, 1573-1580.

Levine, M., and SchorT, C. (1971). Mutations of phage P22 affecting phage DNA synthesis and lysogenization. J. Mol. Biol., 62, 53-64.

Levine, M., and Smith, H. O. (1964). Sequential gene action in the establishment of lysogeny. Science 146, 1581-1582.

Ptashne, M., and Hopkins, N. (1968). The operators controlled by lambda phage repressor. Proc. Nat. Acad. Sci. U. S. 60, 1282-1287.

RAo, R. N. (1968). Bacteriophage P22 controlled exclusion in Salmonella typhimurium. J. Mol. Biol. 35, 607-622.

Robmins, P. W., and Uchids, T. (1962). Determinants of specificity in Salmonella: changes in antigenic structure mediated by bacteriophage. Fed. Proc. Fed. Amer. Soc. Exp. Biol. 21, $702-710$.

SLy, W. S., and Rabideau, K. (1969). Mechanism of $\lambda c_{17} c I$ virulence. J. Mol. Biol. 42, 385-400. 
Smith, H. O., and Levine, M. (1964). Two sequential repressions of $D N A$ synthesis in the establishment of lysogeny by phage P22 and its mutants. Proc. Nat. A cad. Sci. U. S. 52, 356-363.

Taylor, K., Hradecna, Z., and Szybalsiki, W. (1967). Asymmetric distribution of the transcribing regions on the complementary strands of the coliphage $\lambda$ DNA. Proc. Nat. Acad. Sci. U. S. 57, 1618-1625.

Thomas, R., and Bertani, L. E. (1964). On the control of the replication of temperate bacterio- phages superinfecting immune hosts. Virology 24, 241-253.

WALSH, J., and MEYNell, G. G. (1967). The isolation of nonexcluding mutants of phage P22. J. Gen. Virol. 1, 581-582.

Young, B. G., Fukazawa, Y, and Hartman, P. E. (1964). A bacteriophage mutant defective in antigen conversion. Virology 23, 279-283.

ZINDER, N. D. (1958). Lysogenization and superinfection immunity in Salmonella. Virology 5 , 291-326. 